\title{
Suppression of the Richtmyer-Meshkov instability due to a density transition layer at the interface
}

\author{
Takayoshi Sano, 1 , 6 Kazuki Ishigure, ${ }^{1}$ and Fransisco Cobos-Campos ${ }^{2,3}$ \\ ${ }^{1}$ Institute of Laser Engineering, Osaka University, Suita, Osaka 565-0871, Japan \\ ${ }^{2}$ ETSI Industriales, Instituto de Investigaciones Energéticas and CYTEMA, \\ Universidad de Castilla-La Mancha, 13071 Ciudad Real, Spain \\ ${ }^{3}$ Fluid Mechanics Group, Escuela Politécnica Superior, \\ Universidad Carlos III de Madrid, 28911 Leganés (Madrid), Spain \\ (Dated: Jun 4, 2020; accepted for publication in Physical Review E)
}

\begin{abstract}
We have investigated the effects of a smooth transition layer at the contact discontinuity on the growth of the Richtmyer-Meshkov instability (RMI) by hydrodynamic numerical simulations and derived an empirical condition for the suppression of the instability. The transition layer has little influence on the RMI when the thickness $L$ is narrower than the wavelength of an interface modulation $\lambda$. However, if the transition layer becomes broader than $\lambda$, the perturbed velocity associated with the RMI is reduced considerably. The suppression condition is interpreted as the cases that the shock transit time through the transition layer is longer than the sound crossing time of the modulation wavelength. The fluctuation kinetic energy decreases as $L^{-p}$ with $p=2.5$, which indicates that the growth velocity of the RMI decreases in proportion to $L^{-p / 2}$ by the presence of the transition layer. This feature is found to be quite universal and appeared in a wide range of shock-interface interactions.
\end{abstract}

\section{INTRODUCTION}

Interfacial instabilities are of great importance in various plasma phenomena in the universe and laboratory experiments [1, 2]. The Richtmyer-Meshkov instability (RMI) 3, 4] is one of such instabilities that occurs when a planar shock hits a corrugated surface of the contact discontinuity. The RMI has been studied vigorously by the linear theory [5-10], nonlinear analysis [11 15], and laboratory experiments 16 22].

Turbulent mixing excited by the RMI often plays a crucial role associated with plasma explosions in astrophysical objects 23] and the implosion in inertial confinement fusion [24, 25]. Interaction of supernova shocks and inhomogeneous interstellar matters is one of the promising sites of the RMI, which could contribute to the origin of the interstellar turbulence [26] as well as the amplification of magnetic fields [27]. The RMI is recognized as one of the severe obstacles to prevent the ideal implosion in laser fusion plasmas [24, 25]. Drastic symmetry reduction results in inadequate energy gain at the end of the process. Therefore, the mitigation mechanisms of the RMI are paid attention intensely in this field.

There are several effects proposed to stabilize the RMI. The vorticity deposited at the interface just after the incident shock refraction is the driving source of the RMI growth, while the vorticity left in the bulk of the fluids has been proved to be a physical agent that decreases the growth of the contact surface ripple 33, 34]. However, the effect of the bulk vorticity becomes significant only when the shock is sufficiently strong, or the compression is high enough. For the RMI in plasmas, a

\footnotetext{
* sano@ile.osaka-u.ac.jp
}

strong magnetic field can suppress the growth of the RMI when the Alfvén (Mach) number, which is the ratio of the linear growth velocity to the Alfvén speed, is less than unity $28-30]$. However, if the direction of the magnetic field is parallel to the interface but perpendicular to the wavevector of the surface modulation, the Lorentz force hardly works on the RMI. Then, the suppression by the magnetic field in three-dimensional geometry would be difficult so as in the case of the Rayleigh-Taylor instability [31, 32].

In this paper, we focus on the effect of a density transition layer at the interface for the suppression of the RMI. It is well known that the smooth density structure of the interface affects the unstable growth of surface fluctuations. For example, the density stratification at the shear layer of the velocity stabilizes the KelvinHelmholtz instability [31]. The stability condition is given by the Richardson number, which is a function of the density gradient. The Rayleigh-Taylor instability is also affected by the density stratification [24, 35]. The growth rate of the instability decreases dramatically if the scale length of the density structure is longer than the wavelength of the Rayleigh-Taylor mode. As for the RMI, the transition-layer effects have not been much investigated because of the difficulty of the analytical approach [36].

The density transition layer is naturally formed in astrophysical objects and laser plasmas. The molecular clouds in the interstellar medium are modeled by an isothermal self-gravitating sphere, which is the so-called Bonnor-Ebert sphere, where a flat high-density core surrounded by a power-law envelope. The smooth distribution of the density should affect the stability at the shock interaction [37 39]. In laboratory plasmas, the density distribution in the laser ablation layer is verified to mitigate the turbulent-mixing caused by the abla- 
tive Rayleigh-Taylor instability [40, 41]. The exponential density distribution is usually assumed at the material interface in this case. Thus, the RMI with a smooth layer of the density transition would have numerous critical applications.

The goal of this paper is to obtain the suppression condition of RMI due to the existence of the density transition layer by using nonlinear hydrodynamic simulations. The analytical treatment of the RMI is not straightforward when the interface has a non-uniform density profile. In that case, numerical simulations are a powerful tool as the first step to examine such complicated situations and to extract the essence of the physical basis empirically.

The outline of this paper is as follows. In Sec. II the basic equations, initial conditions, and numerical methods are described. Various simulation results are shown in Sec.III to reveal the influence of the transition layer on the growth of the RMI. In Sec. IV] the physical interpretation of our findings is discussed. Then, the suppression condition of the RMI in terms of the thickness of the transition layer is derived. We also remark on an application of our results to laboratory laser plasmas. Finally, the conclusions are summarized in Sec. V.

\section{NUMERICAL METHOD}

To study the nonlinear evolutions of the RMI, the following system equations for inviscid fluids are solved;

$$
\begin{aligned}
\frac{\partial \rho}{\partial t}+\nabla \cdot(\rho \boldsymbol{v}) & =0 \\
\frac{\partial(\rho \boldsymbol{v})}{\partial t}+\nabla \cdot[P \boldsymbol{I}+\rho \boldsymbol{v} \boldsymbol{v}] & =0 \\
\frac{\partial e}{\partial t}+\nabla \cdot[(e+P) \boldsymbol{v}] & =0
\end{aligned}
$$

where $\rho$ and $\boldsymbol{v}$ are the mass density and velocity, respectively, and $e$ is the total energy density per unit volume, $e=P /(\gamma-1)+\rho v^{2} / 2$. The equation of state for the ideal gas is used with the isentropic exponent $\gamma$.

We adopt a single-mode setup for our numerical analysis, which is illustrated by Fig. 11(a). Two fluids with different densities, $\rho_{a 0}$ and $\rho_{b 0}$, are separated by a corrugated interface located at $x=0$. A planar shock propagating through the fluid "b" hits the corrugated interface at $t=0$. Here the $x$ - and $y$-axis are set to be perpendicular and parallel to the shock surface. The incident shock velocity is $-U_{i} \hat{x}$, where $\hat{x}$ is a unit vector. Both the fluids are stationary $\boldsymbol{v}=0$ and have a uniform pressure $P_{0}$ before the shock passage. The sonic Mach number of the incident shock is defined as $M=U_{i} / c_{b 0}$ where $c_{b 0}=\left(\gamma_{b} P_{0} / \rho_{b 0}\right)^{1 / 2}$ is the sound speed of the fluid "b". The physical quantities in the post-shocked region behind the incident shock are calculated from the RankineHugoniot conditions. The interface has an initial corrugation of a sinusoidal form, $x=\psi_{0} \cos (k y)$, where $\psi_{0}$ is

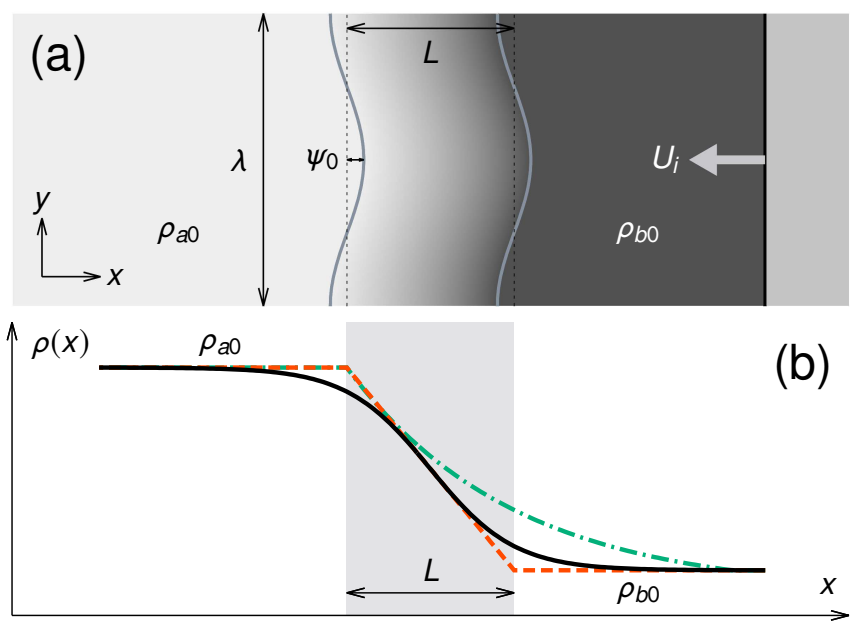

FIG. 1. (a) Initial setup of the single-mode analysis for the RMI. A planar shock hits a corrugated interface between the fluid "a" and "b", where the wavelength of the surface modulation is assumed to be $\lambda$. The characteristic quantities of this system are the incident shock velocity $U_{i}$, the density jump at the interface $\rho_{a 0} / \rho_{b 0}$, and the corrugation amplitude $\psi_{0}$. In this analysis, the density transition layer with a thickness of $L$ is considered. (b) Density distributions of the transition layer adopted in this analysis, which are the tanh-type (black), linear-type (red), and exp-type (green). The transition layer is located at $-L / 2 \leq x \leq L / 2$ initially.

the corrugation amplitude, $k=2 \pi / \lambda$ is the perturbation wavenumber, and $\lambda$ is the wavelength.

Six non-dimensional parameters characterize the initial configuration of the nonlinear single-mode analysis with a transition layer. The Mach number $M$ parameterizes the incident shock velocity. The contact discontinuity is expressed by the density jump $\rho_{a 0} / \rho_{b 0}$ and the ratio of the corrugation amplitude to the wavelength $\psi_{0} / \lambda$. The compressibility indicated by the isentropic exponents $\gamma_{a}$ and $\gamma_{b}$ of each fluid is also an essential element of this system. Besides, we introduce a density transition layer with a finite width of $L$, which provides an additional parameter of $L / \lambda$. In this analysis, we assume a constant value of $\gamma_{a}=\gamma_{b}=5 / 3$ for simplicity. Thus, the other four parameters $\left(M, \rho_{a 0} / \rho_{b 0}, \psi_{0} / \lambda\right.$, and $\left.L / \lambda\right)$ are considered in the following.

Various shapes of the density distribution in the layer could be plausible according to circumstances. As a typical function, we take a hyperbolic-tangent function expressed as

$$
\rho(x)=\frac{\rho_{a 0}+\rho_{b 0}}{2}\left[1-A \tanh \left(\frac{2 x}{L}\right)\right],
$$

where $A=\left(\rho_{a 0}-\rho_{b 0}\right) /\left(\rho_{a 0}+\rho_{b 0}\right)$ is the Atwood number. Linear and exponential distributions are also examined 
for comparison, which are given by

$$
\rho(x)= \begin{cases}\rho_{a 0} & \left(x \leq-\frac{L}{2}\right) \\ \frac{\rho_{a 0}+\rho_{b 0}}{2}\left[1-A\left(\frac{2 x}{L}\right)\right] & \left(|x|<\frac{L}{2}\right) \\ \rho_{b 0} & \left(x \geq \frac{L}{2}\right)\end{cases}
$$

and

$$
\rho(x)=\left\{\begin{array}{ll}
\rho_{a 0} & \left(x \leq-\frac{L}{2}\right) \\
\max \left\{\rho_{a 0} e^{-\frac{1}{L}\left|x+\frac{L}{2}\right|}, \rho_{b 0}\right\} & \left(x>-\frac{L}{2}\right)
\end{array} .\right.
$$

While the thickness of the transition layer is well defined by $L$ in the hyperbolic-tangent and linear distributions, the effective thickness in the exponential-type depends on the combination of the scale length $L$ and density jump $\rho_{a 0} / \rho_{b 0}$.

There are several formulas suggested evaluating the linear growth velocity of the RMI theoretically. The linear growth with time, not exponential, is one of the unique characteristics of the RMI. Another feature of the RMI is that it occurs in both cases of light-to-heavy $\left(\rho_{a 0} / \rho_{b 0}>1\right)$ and heavy-to-light $\left(\rho_{a 0} / \rho_{b 0}<1\right)$ configurations.

Richtmyer 3] was the first to study the problem of a planar shock crossing the corrugated boundary between two fluids, and proposed a generalization of the RayleighTaylor formula as the growth velocity $\partial \psi / \partial t=k v^{*} A^{*} \psi_{0}^{*}$, where $v^{*}$ and $\psi_{0}^{*}=\psi_{0}\left(1-v^{*} / U_{i}\right)$ are the zero-order velocity and the amplitude of the contact surface just after the shock passage, respectively. The Atwood number $A^{*}=\left(\rho_{a}^{*}-\rho_{b}^{*}\right) /\left(\rho_{a}^{*}+\rho_{b}^{*}\right)$ is defined by the densities at both sides of the post-shocked interface. Then, Meyer and Blewett [5] observed that the Ricthmyer prescription should be modified using an averaged value between the pre- and post-shocked interface amplitude, i.e., $\partial \psi / \partial t=k v^{*} A^{*}\left(\psi_{0}+\psi_{0}^{*}\right) / 2$, in order to obtain agreement between the numerical solution and the linear theory. A similar heuristic approach was also proposed by Vandenboomgaerde et al. [10]. Unfortunately, these empirical prescriptions are likely to fail for high compressions [10].

Further linear theories of the RMI have been done in the form of series expansions in terms of inverse powers of the Laplace variable [42, in powers of the time [43], or in terms of the Bessel functions [8, 9, 33, 34]. In particular, the asymptotic growth velocity for both shock- and rarefaction-reflected cases is calculated with the following expression derived by Wouchuk and Nishihara [9]:

$$
v_{\mathrm{wn}}=\frac{-\rho_{a}^{*} \delta v_{y a}^{*}+\rho_{b}^{*} \delta v_{y b}^{*}}{\rho_{a}^{*}+\rho_{b}^{*}}+\frac{\rho_{a}^{*} F_{a}-\rho_{b}^{*} F_{b}}{\rho_{a}^{*}+\rho_{b}^{*}},
$$

where $\delta v_{y a}^{*}$ and $\delta v_{y b}^{*}$ are the initial tangential velocities at both sides of the contact surface. The quantities $F_{a}$ and $F_{b}$ represent the sonic interaction between the contact surface and the transmitted and reflected wavefront, respectively, which are proportional to the amount of vorticity left behind the wavefronts in the bulk of each fluid. For the case when a rarefaction is reflected, no vorticity is created in the expanded fluid, i.e., $F_{b}=0$.

The Wouchuk-Nishihara (WN) formula is rigorously deduced from linearized two-dimensional Euler equations after two wavefronts have separated away from the interface. The growth velocity given by Eq. (7) is exact within the limits of linear theory and inviscid flow. It is valid for any initial configuration, and every element can be analytically calculated from the pre-shocked parameters [33, 34, 44, 45]. The first term of the right-hand side of Eq. (77) is due to the instantaneous deposition of the vorticity at the interface just after the shock interaction. On the other hand, the second term represents the interaction between the contact surface and the wavefronts. It becomes non-negligible for stronger shocks or highly compressible fluids, and typically has the opposite sign to the first term. The negative growth velocity stands for the phase reversal that could occur the rarefaction-reflected cases. Throughout our analysis, the $\mathrm{WN}$ formula $v_{\text {wn }}$ is used as the typical velocity of the RMI growth for a given set of the parameters $\left(M, \rho_{a 0} / \rho_{b 0}\right.$, and $\left.\psi_{0} / \lambda\right)$.

We solve the system equations (11)-(3) in two-dimension $(x, y)$ in the Cartesian coordinate system by using a conservative Godunov scheme with the second-order accuracy in space and time [46, 47]. The exact solutions of the Riemann problem at each grid boundary are used in the flux calculations for time integration of the variables [48]. The scheme includes an additional numerical diffusion in the direction tangential to the shock surface in order to care for the carbuncle instability [49]. A periodic boundary condition is used in the $y$-direction, and an outflow boundary condition is adopted in the $x$-direction. The size of the computational box in the $y$-direction is always set to be $L_{y}=\lambda$. The choice of $L_{x}$, on the other hand, depends on the initial parameters. The $x$-length is taken to be sufficiently extensive so that both of the transmitted shock and reflected shock (or rarefaction) never reach the edge of the computational domain in all the runs.

Most of the calculations are performed with a standard resolution of $\Delta_{x}=\Delta_{y}=\lambda / 256$ unless otherwise stated. The physical quantities are normalized by the initial density and sound speed of the fluid "b", $\rho_{b 0}=1$ and $c_{b 0}=1$, and the wavelength of the surface modulation $\lambda=1$. The sound crossing time of the wavelength is also unity in our normalization, $\lambda / c_{b 0}=1$.

\section{NUMERICAL RESULTS}

First, we look at the difference in the density distribution caused by the existence of a transition layer. The growth of the RMI is usually recognized by the mushroom-shaped elongation of the density interface. Figure 2 shows the contour lines of the density at the nonlinear regime of the RMI growth. The horizontal axis $x$ is converted to a frame moving with the 


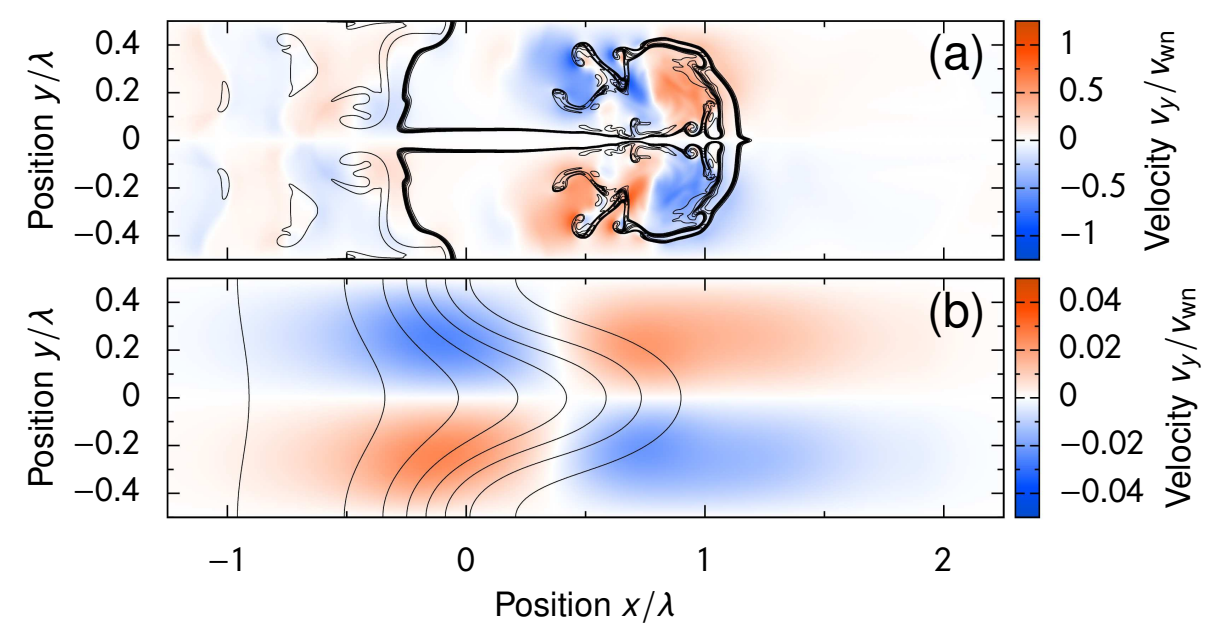

FIG. 2. Spatial distributions of the density $\rho$ and the tangential velocity $v_{y}$ at the nonlinear regime of the RMI growth $k v_{\mathrm{wn}} t=15$ for the cases of (a) $L / \lambda=0.03$ and (b) $L / \lambda=3$. The other parameters are identical for both cases, which are $M=2, \rho_{a 0} / \rho_{b 0}=10$, and $\psi_{0} / \lambda=0.1$. The tanh-type transition layer is assumed. The density distribution is depicted by the contour curves at the levels from $\rho / \rho_{b 0}=6$ to 27 . The color map denotes the tangential velocity normalized by the growth velocity of the WN model $v_{y} / v_{\mathrm{wn}}$. The color-bar range of the velocity in (b) is $1 / 25$ of that in (a).

contact-discontinuity velocity $v^{*}$ after the shock interaction. The initial parameters in this fiducial run are the Mach number $M=2$, the density jump $\rho_{a 0} / \rho_{b 0}=10$, and the modulation amplitude $\psi_{0} / \lambda=0.1$. The function of the density transition layer is the hyperbolic tangent given by Eq. (4). The snapshot is taken at $k v_{\mathrm{wn}} t=15$, where $\left(k v_{\mathrm{wn}}\right)^{-1}$ is the unit timescale of the RMI. The linear growth velocity of the WN model $v_{\text {wn }}$ is evaluated from Eq. (77). For the fiducial parameters, $v_{\mathrm{wn}} / c_{b 0}=0.20651$, so that the RMI timescale corresponds to slightly shorter than the initial sound crossing time, $\left(k v_{\mathrm{wn}}\right)^{-1} \sim 0.77\left(\lambda / c_{b 0}\right)$.

For a narrow transition case of $L / \lambda=0.03$ [Fig. 2(a)], the RMI growth is nearly identical to the case with a sharp boundary case $(L=0)$. The width of the mixing layer defined from the spike top to bubble bottom exceeds the modulation wavelength of $\lambda$. On the other hand, the deformation of the interface is significantly reduced when the transition layer becomes comparable to $\lambda$. Figure 2(b) shows the density contours for a case of $L / \lambda=3$, in which the other parameters are the same as those of Fig. 2(a). Although the location of the interface cannot be defined uniquely for this case, the density contours are rather smooth compared to those in Fig. 2(a). The fluctuation amplitude of the contour lines is at most a few times larger than the initial corrugation amplitude of $\psi_{0} / \lambda=0.1$. Thus, the transition layer indeed mitigates the growth of the RMI. The enhancement of the modulation in the density structure is severely suppressed.

Because the RMI growth is tightly connected to the tangential velocity induced by shock interaction with a corrugated interface, we focus on the $y$-component of the perturbed velocity in our simulations. Note that $v_{y}$ is nothing everywhere before the shock passage since we consider homogeneous initial flow. In Figs. 2(a) and2(b), the tangential velocity $v_{y}$ normalized by $v_{\mathrm{wn}}$ is depicted by colors for each case.

The tangential velocity is of the order of $v_{\mathrm{wn}}$ when $L / \lambda=0.03$ [Fig. 2(a)]. At the time of the snapshot $k v_{\text {wn }} t=15$, the fastest velocity is localized at the roll-up region of the mushroom shape. The mixing-layer width due to the RMI motions is still growing even at this nonlinear phase. By contrast, the generation of $v_{y}$ is weakened by more than an order of magnitude in the broad transition case of $L / \lambda=3$ [Fig. 2(b)]. The color range of the tangential velocity in Fig. 2(b) is about $1 / 25$ of that in Fig. 2(a). Weak tangential shear is deposited in the middle of the transition layer, where the most considerable distortion of the density contour is observed. The location of the maximum vorticity would be related to the largest gradient of the density.

The tangential velocity is a good indicator of the activity of the RMI. The time evolutions of the maximum value of $\left|\rho v_{y}\right|$ for two cases in Fig. 2] are shown by the solid curves in Fig. 3. The maximum momentum is divided by $\left(\rho_{a}^{*}+\rho_{b}^{*}\right) v_{\mathrm{wn}}$ so that the vertical axis approximately indicates the effective growth velocity relative to the original $v_{\mathrm{wn}}$ of the $\mathrm{WN}$ model. When the transition layer is narrow $(L / \lambda=0.03)$, the growth velocity appears instantaneously at $t=0$ and keeps nearly constant around $\sim v_{\mathrm{wn}}$. However, the growth velocity in the broader transition case $(L / \lambda=3)$ increases gradually during the shock travels in the smooth density distribution. The peak value is much lower than the linear-theory prediction for the discontinuous case. Since the density changes continuously in the transition layer, the shock pressure at the downstream, or the shock strength, is weakened compared to the discontinuous case. Such an effectively weaker shock causes a significant reduction of 

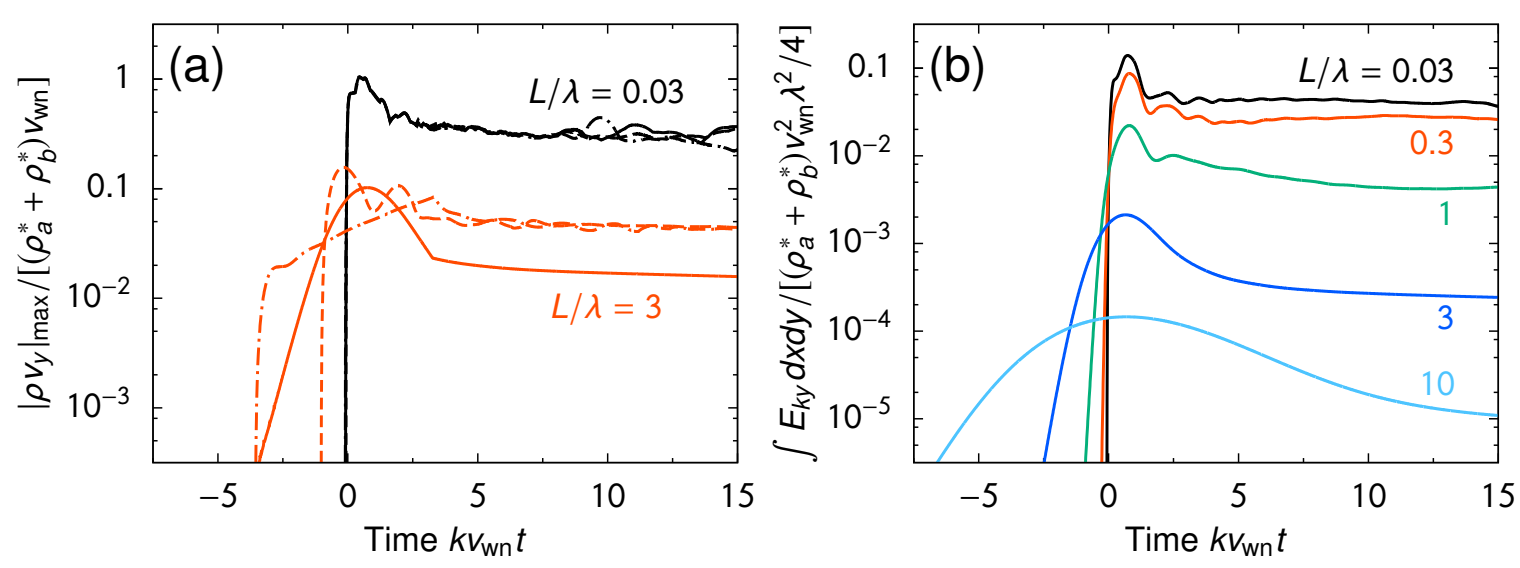

FIG. 3. (a) Time evolutions of the maximum tangential momentum $\left|\rho v_{y}\right|$ in the entire area of the computational domain for the cases of $L / \lambda=0.03$ (black) and $L / \lambda=3$ (red). The other parameters are identical to the fiducial runs shown in Fig. 2 . The dependence on the density function is also indicated in this figure by the different line types. The results of the tanh-type, liner-type, and exp-type are depicted by the solid, dashed, and dot-dashed curves, respectively. The time in the horizontal axis is given in the unit of the RMI timescale $\left(k v_{\mathrm{wn}}\right)^{-1}$. (b) Time histories of the kinetic energy defined by the tangential velocity $E_{k y} \equiv \rho v_{y}^{2} / 2$ integrated over the entire domain. The thickness of the tanh-type transition layer is labeled for each curve. The other parameters are the same as those of the runs shown in (a).
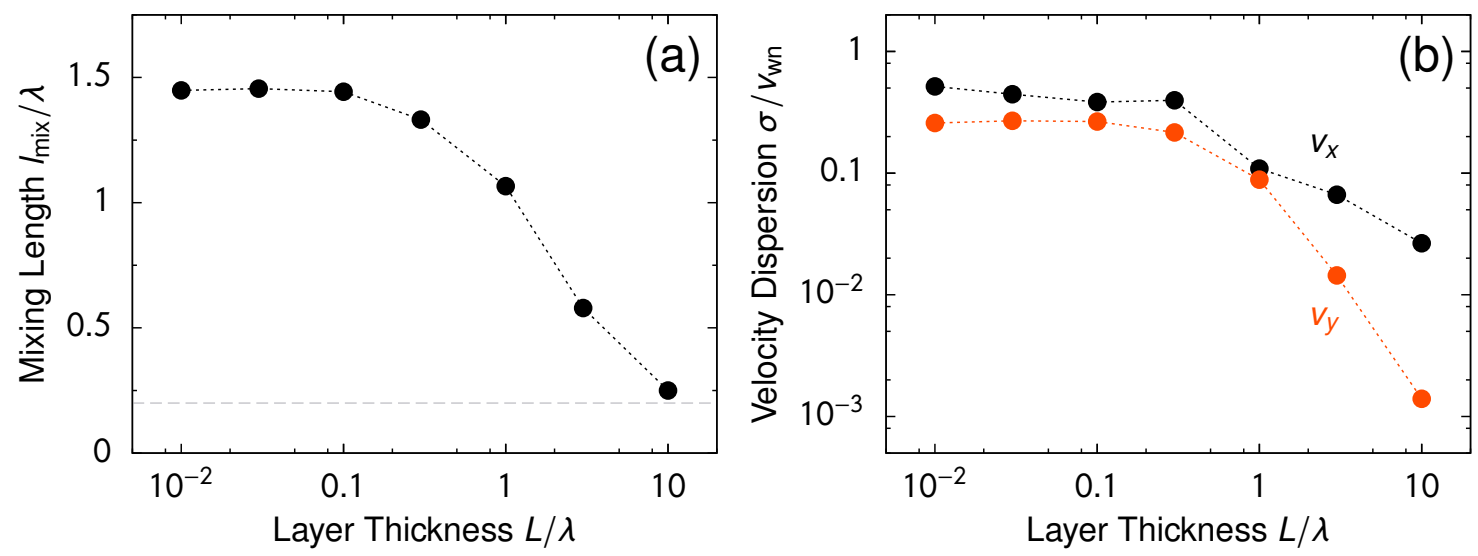

FIG. 4. (a) Dependence of the mixing length $l_{\text {mix }}$ caused by the RMI growth on the thickness of the transition layer. The mixing length is defined by the distance from the spike top to the bubble bottom, which is evaluated through the tracer particle positions at $k v_{\mathrm{wn}} t=15$. The simulation parameters are the same as those in the fiducial run except for $L$. The gray dashed line denotes the initial amplitude of $2 \psi_{0} / \lambda=0.2$. (b) Standard deviation $\sigma$ of the interface velocities $v_{x}$ and $v_{y}$ in terms of the layer thickness $L$. The interface velocities are taken at $k v_{\mathrm{wn}} t=15$ in the same runs as in (a).

the growth velocity of RMI in the linear phase [44, 45].

The suppression effect due to non-zero $L$ seems to have little dependence on the functional form of the density in the transition layer. The time histories of the maximum tangential momentum in the linear-type [Eq. [5]] and exponential-type [Eq. (6)] distribution are also plotted in Fig. 3(a) by the dashed and dot-dashed curves, respectively. The difference in the density function is negligible if the layer is much narrower than the modulation wavelength. Huge decrease of the growth velocity $v_{y}$ is observed in all the cases of $L / \lambda=3$, where the peak and asymptotic velocities are quite similar among the different function cases.

The fluctuation kinetic energy at the nonlinear regime of RMI may be a useful quantity to evaluate the sup- pression effect by the transition layer. Figure 3(b) shows the evolutions of the perturbed kinetic energy defined by $E_{k y} \equiv \rho v_{y}^{2} / 2$ integrated over the entire region. The initial parameters are the same as in Fig. 2 except for the thickness of the transition layer $L$. As can be seen, the fluctuation kinetic energy decreases drastically if $L / \lambda \gtrsim 1$. When $L / \lambda=10$, for example, the integrated $E_{k y}$ is reduced by about three orders of magnitude compared to the sharp transition case $L / \lambda \sim 0$. In the $x$-direction, it is hard to define the perturbed velocity because the unperturbed distribution is also time-dependent. However, the $x$-component of the perturbed velocity must be comparable to that in the $y$-direction in the RMI motions. Thus we believe that Figure 3(b) is representing the fluctuation kinetic energy driven by the RMI properly. 
The $L$ dependence shown by Figs. 3(a) and 3(b) are obtained by the simulations with the resolution of $\Delta_{x}=$ $\Delta_{y}=\lambda / 512$. These results are found to be unaffected by the numerical resolution, which is confirmed by identical calculations with different grid sizes of $\lambda / 1024$ and $\lambda / 256$. For the case of $L / \lambda=0.01$, the transition layer is captured by only five grids, so that its result would be regarded as that for the discontinuous case.

Lagrangian tracer particles are often used to pursue the evolution of the interface shape and velocity distribution for the case of a sharp density jump. Here we apply this method even for the cases with a finite transition layer. The tracer particles are set initially at the center of the transition layer, that is, along a line given by $x=\psi_{0} \cos (k y)$ for all cases. Mixing length $l_{\text {mix }}$ is calculated from the difference between the maximum and minimum values of the $x$-coordinate among these particles. Figure 4(a) shows the characteristic mixing length by the RMI growth at $k v_{\mathrm{wn}} t=15$ for various runs with different thickness of the transition layer. The modulation amplitude at the nonlinear stage of the RMI has an apparent dependence on $L$. For the case of $L / \lambda=10$, the mixing length is almost the same as the initial modulation amplitude. This fact indicates the severe suppression of the RMI due to the transition layer, which is consistent with the result shown in Figs. 2 and 3 .

The perturbed velocity of the interface is inferred from the tracer particle velocities. We can evaluate the standard deviation $\sigma$ of the interface velocity (or the velocity dispersion), which is shown in Fig. 4(b). Both components of $v_{x}$ and $v_{y}$ exhibit the same trend of the $L$ dependence, as seen in Fig. 世(a). Since the average velocity of $v_{y}$ is zero, the velocity dispersion $\sigma$ is identical to the root-mean-square of $v_{y}$. If the thickness of the transition layer is negligible compared to the modulation wavelength, the perturbed velocity is comparable to the growth velocity of the WN model. Although the velocity dispersion of $v_{x}$ in this limit is slightly larger than that of $v_{y}$, the difference is no more than double. On the other hand, the unperturbed profile of $v_{x}$ in the transition layer depends on the position $x$. Thus, the velocity dispersion may tend to be larger than the perturbed component alone, as the transition layer becomes thick. This is another reason why we concentrate the $y$-component of the perturbed velocity.

It is found that the dependence of the RMI suppression on $L / \lambda$ is quite robust and valid for a wide range of the initial parameters. The fluctuation kinetic energy measured at $k\left|v_{\mathrm{wn}}\right| t=15$ for various cases listed in Table $\prod$ are shown all together in Fig. 5 Normalization of the kinetic energy in this diagram is to divide by $\left(\rho_{a}^{*}+\rho_{b}^{*}\right) v_{\mathrm{wn}}^{2} \lambda^{2} / 4$ that is proportional to $v_{\mathrm{wn}}^{2}$. It should be noted that the dimensionless linear growth velocity $v_{\mathrm{wn}} /\left(k \psi_{0} U_{i}\right)$ is determined by $M, \rho_{a 0} / \rho_{b 0}$, and $\gamma_{a}=\gamma_{b}=5 / 3$ in our system. For example, a higher Mach number gives a faster growth velocity so that the normalization factor is larger for the higher $M$ case. The growth velocity $v_{\text {wn }}$ for each case is also listed in Table 【

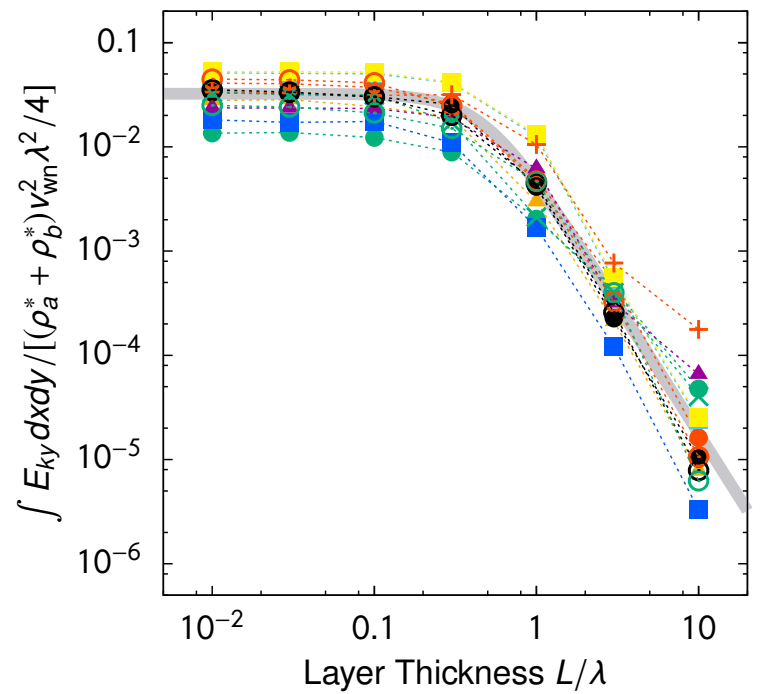

FIG. 5. Dependence of the integrated kinetic energy $\int E_{k y} d x d y$ measured at the nonlinear regime $k\left|v_{\mathrm{wn}}\right| t=15$ on the thickness of the transition layer $L$. Various parameter runs listed in Table \are plotted with different marks. The meaning of each mark is described in the last column of Table I The gray thick curve is the fitted function of all the data, which is proportional to $\left[1+(q L / \lambda)^{p}\right]^{-1}$ with $q=2.11$ and $p=2.46$.

All the data points in Fig. 5 exhibit a single trend on $L / \lambda$, even though the vast parameter range of many orders of magnitude are examined here. The fiducial set of the parameters are chosen as $M=2, \rho_{a 0} / \rho_{b 0}=10$, $\psi_{0} / \lambda=0.1$, and tanh-type function. Then we examine the dependence of the transition function (linear- and exp-type), the Mach number $(M=1.2,10$, and 100), the density jump for shock-reflected cases $\left(\rho_{a 0} / \rho_{b 0}=3\right.$ and $100)$ and for rarefaction-reflected cases $\left(\rho_{a 0} / \rho_{b 0}=0.3\right.$, 0.1 , and 0.01$)$, and the modulation amplitude $\left(\psi_{0} / \lambda=\right.$ 0.03 and 0.3 ). For each parameter case, we perform seven runs with different widths of the transition layer in a range from $L / \lambda=0.01$ to 10 to identify the dependence.

When $L / \lambda \lesssim 1$, the fluctuation kinetic energy is flat and almost identical to that in the discontinuous limit $L \rightarrow 0$. On the other hand, if $L / \lambda$ becomes larger than unity, the kinetic energy decreases with a power law. The dependence could be fitted by a function proportional to $\left[1+(q L / \lambda)^{p}\right]^{-1}$ with two fitting parameters $q$ and $p$. The fitted results for $q$ and $p$ are listed in Table \ for each parameter case. The average values of all the cases are $q=2.11 \pm 0.35$ and $p=2.46 \pm 0.17$, which is drawn by the gray thick curve in Fig. 5 . Thus, the influence of the transition layer begins to appear when $L \gtrsim \lambda / 2$. In the limit of $L / \lambda \gg 1$, the fluctuation kinetic drops in proportion to $L^{-5 / 2}$, which means the growth velocity has a power-law dependence of $L^{-5 / 4}$ approximately. 
TABLE I. A list of the initial conditions for the simulations shown in Fig. 5. The key parameters are the Mach number of the incident shock $M$, the density jump at the contact discontinuity $\rho_{a 0} / \rho_{b 0}$, and the modulation amplitude relative to the wavelength of $\psi_{0} / \lambda$. Three types of the transition layer (tanh, linear, and exp) are considered. The linear growth velocity $v_{\mathrm{wn}}$ of the RMI is calculated based on the WN model. The obtained kinetic energy is fitted by $\left[1+(q L / \lambda)^{p}\right]^{-1}$ as a function of $L / \lambda$, and the fitted results for $q$ and $p$ are listed for each parameter set in the table. A numerical factor $\zeta$ for each run is calculated by solving appropriate Riemann problems. The last column is the mark of the plot in Fig. 5 for each case.

\begin{tabular}{cccccccccc}
\hline \hline \multirow{2}{*}{$M$} & $\frac{\rho_{a 0}}{\rho_{b 0}}$ & $\frac{\psi_{0}}{\lambda}$ & transition & $\frac{v_{\mathrm{wn}}}{k \psi_{0} U_{i}}$ & $\frac{v_{\mathrm{wn}}}{c_{b 0}}$ & $q$ & $p$ & $\zeta$ & mark \\
\hline 2 & 10 & 0.1 & $\tanh$ & 0.16433 & 0.20651 & 2.08 & 2.66 & 1.8 & black filled circle \\
2 & 10 & 0.1 & $\operatorname{linear}$ & 0.16433 & 0.20651 & 1.87 & 1.89 & 1.8 & red plus \\
2 & 10 & 0.1 & exp & 0.16433 & 0.20651 & 3.90 & 1.84 & 1.8 & green cross \\
1.2 & 10 & 0.1 & $\tanh$ & 0.082788 & 0.062421 & 2.19 & 2.72 & 1.6 & blue square \\
10 & 10 & 0.1 & $\tanh$ & 0.18589 & 1.1680 & 1.58 & 2.79 & 2.0 & cyan square \\
100 & 10 & 0.1 & $\tanh$ & 0.18639 & 11.711 & 1.56 & 2.80 & 2.0 & yellow square \\
2 & 3 & 0.1 & $\tanh$ & 0.13365 & 0.16795 & 2.01 & 2.53 & 1.6 & red filled circle \\
2 & 100 & 0.1 & $\tanh$ & 0.081918 & 0.10294 & 2.56 & 1.73 & 2.0 & green filled circle \\
2 & 0.3 & 0.1 & $\tanh$ & -0.20979 & -0.26364 & 2.17 & 2.65 & 1.1 & red open circle \\
2 & 0.1 & 0.1 & $\tanh$ & -0.35302 & -0.44362 & 1.92 & 2.77 & 0.80 & black open circle \\
2 & 0.01 & 0.1 & $\tanh$ & -0.44584 & -0.56026 & 1.39 & 3.01 & 0.34 & green open circle \\
2 & 10 & 0.03 & $\tanh$ & 0.16433 & 0.061953 & 2.16 & 2.63 & 1.8 & orange triangle \\
2 & 10 & 0.3 & $\tanh$ & 0.16433 & 0.61953 & 2.09 & 2.03 & 1.8 & purple triangle \\
\hline \hline
\end{tabular}

\section{DISCUSSION}

Our numerical results suggest that the growth of the RMI is clearly mitigated when the thickness of the transition layer becomes comparable to the modulation wavelength. Here we will consider the physical basis for this outcome.

The competition of two timescales reasonably evaluates the effect of the transition layer. One of those timescales is the transit time $\tau_{\operatorname{tr}}$ of the incident shock to pass through the transition layer. It is given by $\tau_{\text {tr }} \equiv L /\left\langle U_{i}\right\rangle$ where $\left\langle U_{i}\right\rangle$ is the averaged shock velocity in the transition layer. The other one is the stabilizing time $\tau_{\text {st }}$ for the pressure fluctuations by sound waves, which is expressed as $\tau_{\text {st }} \equiv \lambda /\left\langle c^{*}\right\rangle$. Here $\left\langle c^{*}\right\rangle$ is the averaged sound speed at the downstream of the shock.

When the transit time is shorter than the stabilizing time, the transition layer has little effect on the growth of the RMI. However, if $\tau_{\text {st }} \lesssim \tau_{\text {tr }}$, the RMI growth should be modified by the presence of the transition layer. Then the suppression condition is given by $L \gtrsim \zeta \lambda$, where $\zeta \equiv$ $\left\langle U_{i}\right\rangle /\left\langle c^{*}\right\rangle$. We can guess the size of $\zeta$ assuming $\left\langle U_{i}\right\rangle \sim$ $M c_{b 0}$ and $\left\langle c^{*}\right\rangle \sim\left(c_{a}^{*}+c_{b}^{*}\right) / 2$, where $c_{a}^{*}\left(c_{b}^{*}\right)$ is the sound speed of the post-shocked fluid "a" ("b") at the interface for the $L=0$ case. It turns out by solving appropriate Riemann problems that $\zeta$ is of the order of unity for most of the cases we examined (see Table I). Therefore the suppression condition is approximately given by

$$
\frac{\tau_{\text {tr }}}{\tau_{\text {st }}} \sim \frac{L}{\lambda} \gtrsim 1
$$

which is consistent with our numerical results. Interestingly, this interpretation is independent of the density

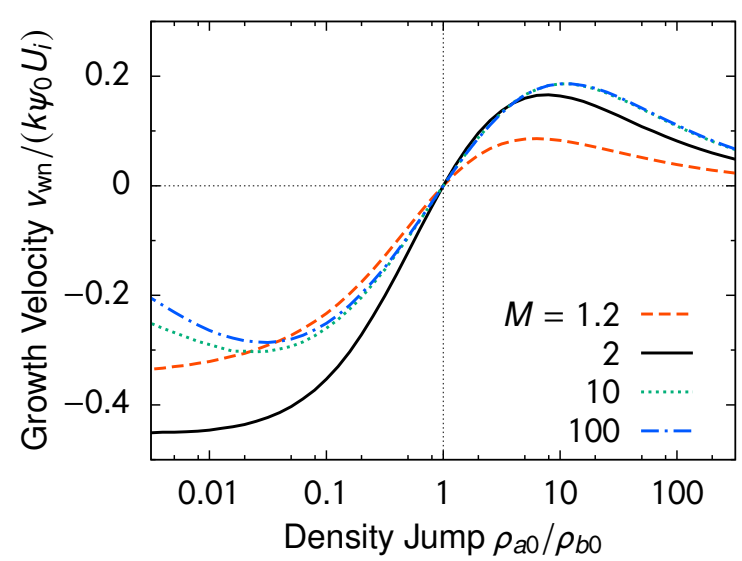

FIG. 6. The linear growth velocity of the WN formula calculated from Eq. (7) shown as a function of the density jump $\rho_{a 0} / \rho_{b 0}$ for various Mach number cases; $M=1.2$ (red dashed), 2 (black solid), 10 (green dotted), and 100 (blue dotdashed). The growth velocity is normalized by $k \psi_{0} U_{i}$. For the isentropic exponent, $\gamma_{a}=\gamma_{b}=5 / 3$ is assumed in all cases.

gradient, which brings a difference from the stability condition for the Kelvin-Helmholtz instability [31].

If the transition layer is broader than the wavelength of the interface modulation, the kinetic energy of the RMI motions decreases with the power of $L^{-p}$ where $p \sim 2.5$. This result is equivalent that the growth velocity of the RMI is reduced in proportion to $L^{-p / 2}$ when $L \gtrsim \lambda$. There might be several reasons for the suppression of the RMI due to the density transition layer.

If $\tau_{\mathrm{st}}$ is shorter than $\tau_{\mathrm{tr}}$, the density difference felt by 
the incident shock is smaller than the discontinuous case, $\Delta \rho_{0} \equiv\left|\rho_{a 0}-\rho_{b 0}\right|$. The effective density difference is then estimated as $\Delta \rho_{\text {eff }} \sim \Delta \rho_{0} \lambda / L$ assuming the linear density gradient. In a range of $0.01 \leq \rho_{a 0} / \rho_{b 0} \leq 100$ examined in our analysis, the growth velocity has a complicated dependence on the density jump [50]. Figure [6 shows the growth velocity of the WN model as a function of the density jump $\rho_{a 0} / \rho_{b 0}$ for different Mach number cases. In this figure, we assume $\gamma_{a}=\gamma_{b}=5 / 3$ for the isentropic exponent. For the case of $\gamma_{a}=\gamma_{b}$, the growth velocity of the RMI must be zero when $\rho_{a 0} / \rho_{b 0}=1$.

In the limit of the small density difference $\Delta \rho_{0} / \rho_{b 0} \ll$ 1 , the asymptotic linear growth velocity has a scaling law of the form [33, 34]

$$
\frac{\left|v_{\mathrm{wn}}\right|}{k \psi_{0} U_{i}} \approx c_{1}^{s, r} \frac{\Delta \rho_{0}}{\rho_{b 0}}+O\left(\frac{\Delta \rho_{0}^{2}}{\rho_{b 0}^{2}}\right),
$$

where a coefficient $c_{1}^{s, r}(>0)$ has different expressions for shock- or rarefaction-reflected cases (see Appendix). Replacing $\Delta \rho_{0}$ in Eq. (9) with the effective density difference $\Delta \rho_{\text {eff }}$, the growth velocity in the limit of $L / \lambda \gg 1$ is obtained by

$$
\frac{\left|v_{\mathrm{wn}}\right|}{c_{b 0}} \sim 2 \pi c_{1}^{s, r} M \frac{\Delta \rho_{0}}{\rho_{b 0}}\left(\frac{\psi_{0}}{\lambda}\right)\left(\frac{L}{\lambda}\right)^{-1} .
$$

This relation suggests that the mitigation of the RMI is larger as the transition layer becomes broader, and which implies the qualitative coincidence with the numerical results shown by Fig. 5

The physical reason behind the RMI suppression is that as the transmitted shock advances through the transition layer, its ripple decreases. In the RMI, perturbations are generated as the result of the conservation of the tangential momentum across the fronts. Thus, the smaller the shock ripple is, the weaker the perturbations are. In the end, the mixing motions developed by the RMI is weakened due to the transition layer. Likewise, weakened shock strength because of the smooth density gradient promotes the suppression and affects the quantitative dependence of the growth velocity. Thus, the index $p \sim 2.5$ might be determined by the combination of multiple origins, although the value seems to be valid in a wide range of parameters. Analytic study on the transition-layer effects would be challenging future work but inevitable for further understanding.

In this work, we assume the isentropic exponent is constant everywhere $\gamma_{a}=\gamma_{b}=5 / 3$. The suppression due to the transition layer is affected by $\gamma$ through the stabilizing time $\tau_{\text {st }}$. Then, for a given thickness of $L$, the stiffer equation of state would be easier to reduce the perturbed velocity of the RMI.

Lastly, we consider the application of our results for laboratory laser plasmas. The existence of a laser ablation plasma at a target surface could play a role as a transition layer during shock interaction. Exponential distribution of the density is often assumed for the ablation plasmas. Suppose a case of $\rho_{a 0} / \rho_{b 0}=0.01$, for

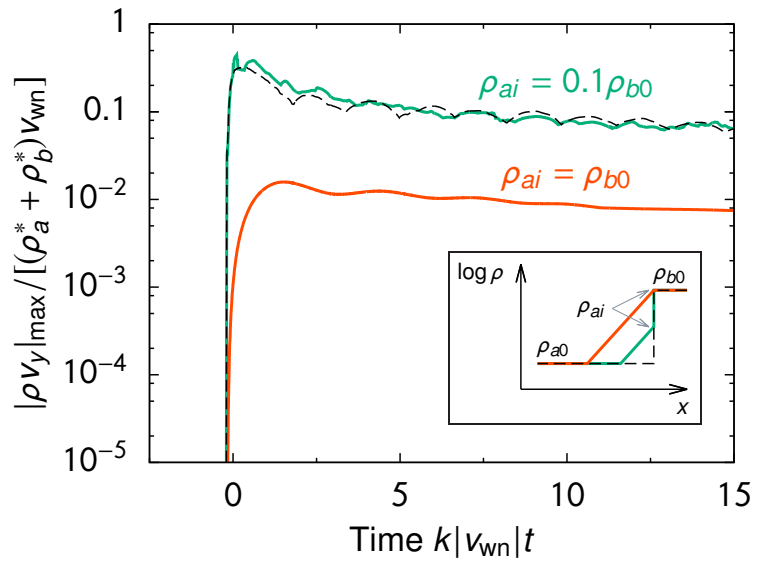

FIG. 7. Time evolutions of the maximum tangential velocity in the exponential transition layer for different interface density of $\rho_{a i}=\rho_{b 0}$ (red) and $\rho_{a i}=0.1 \rho_{b 0}$ (green). The density jump is $\rho_{a 0} / \rho_{b 0}=0.01$ and the scale length is assumed as $L / \lambda=10$ for both cases. The other parameters are the same as in the fiducial run $\left(M=2\right.$ and $\left.\psi_{0} / \lambda=0.1\right)$. The time profile in the corresponding discontinuous case of $L=0$ is also shown by the black dashed curve. (inset) Initial density profiles in the logarithmic scale for two cases of $\rho_{a i}=\rho_{b 0}$ (red) and $\rho_{a i}=0.1 \rho_{b 0}$ (green).

instance, we need to decide the interface density $\rho_{a i}$ as for the edge value of the exponential distribution. The interface density $\rho_{a i}$ would depend on the details of the target density, laser intensity, and pulse shape so that it has substantial ambiguity.

Then, we perform demonstrative calculations with different $\rho_{a i}$ in the suppression case of $L / \lambda=10$, which is shown in Fig. 7 This figure shows the maximum of the tangential momentum $\left|\rho v_{y}\right|$ searched from the entire domain for a given time. The behaviors of the RMI are profoundly affected by the assumption of $\rho_{a i}$. Even if ten percent of the density jump exists, i.e., $\rho_{a i}=0.1 \rho_{b 0}$, the RMI growth in the $L / \lambda=10$ case becomes as active as the no-transition case. In order to benefit from the stabilization by the transition layer, the density distribution should be continuous from $\rho_{b 0}$ to $\rho_{a 0}$. This could be crucial in designing laser experiments for inertial confinement fusion, where the elimination of the interfacial instabilities is really demanded [24, 25].

\section{CONCLUSIONS}

We have investigated the role of the density transition layer on the growth of the RMI using two-dimensional hydrodynamic simulations. Although three-dimensional evolutions of hydrodynamic instabilities are essential in many cases, the two-dimensional study is still important to understand the physics behind it. A universal condition for the suppression of RMI due to the transition layer has been obtained successfully through the systematic parameter study. If the transition layer is narrower 
than the wavelength of the surface modulation, the effect on the RMI is ignorable. However, the RMI growth is severely reduced when the thickness of the transition layer exceeds the modulation wavelength. The obtained threshold condition, $L \gtrsim \lambda$, can be explained by the comparison between the shock-transit time through the transition layer and the stabilizing time of the pressure fluctuations by sound waves. This simple criterion will be useful to evaluate the importance of the RMI in various situations, such as interstellar shock waves in astrophysical phenomena and laser-driven shocks in inertial confinement fusion experiments.

An exhaustive analytic study on the transition-layer effects should be necessary. Nonetheless, the inclusion of compressibility effects and double reflection of reflected waves makes the calculations extremely cumbersome, and it is proposed as future work.

\section{ACKNOWLEDGMENTS}

We thank K. Mima, K. Nishihara, H. Sakagami, Y. Sentoku and J. G. Wouchuk for useful discussions and encouragement. Computations were carried out on SXACE Lite at the Institute of Laser Engineering, Osaka University. This work was partly performed un- der the joint research project of the Institute of Laser Engineering, Osaka University. This research was supported by JSPS KAKENHI Grant No. JP26287147 and No. JP19KK0072, JSPS Core-to-Core Program, B. AsiaAfrica Science Platforms No. JPJSCCB20190003, and MEXT Quantum Leap Flagship Program Grant No. JPMXS0118067246. F.C.-C. has received support from MINECO under Grant No. ENE2016-75703-R, from JCCM Grant No. SBPLY/17/180501/000264, and from BBVA Foundation Leonardo Grant No. 2019/00570/001.

\section{Appendix: The growth velocity of the RMI in the limit of small density jump}

In the limit of small pre-shocked density jump, the asymptotic growth velocity $v_{\mathrm{wn}}$ is given by the scaling laws provided by Eq. (56) in [33] and Eq. (167) in [34], for the shock- and rarefaction-reflected cases, respectively. Assuming $\gamma_{a}=\gamma_{b}=\gamma$, the constant term $c_{0}^{s, r}$ of the scaling laws becomes zero, and, hence, the growth velocity is proportional to the density difference as indicated in Eq. (91). The first term coefficients $c_{1}^{s}$ and $c_{1}^{r}$ are approximately given by the following expressions as a function of the Mach number $M$ and the isentropic exponent $\gamma$.

For the shock-reflected case:

$$
\begin{aligned}
c_{1}^{s}(M, \gamma) & =-\frac{\pi_{3}^{2}\left(M^{2}-1\right)}{\left(\pi_{1}+2 \pi_{2} \pi_{3} \pi_{4}\right)(\gamma+1)^{2} M^{2}\left[2(\gamma-2) M^{2}+\gamma-1\right]\left[(2 \gamma-1) M^{4}+2 M^{2}+1\right]} \\
& \times\left(\pi_{1}\left[\pi_{3} \pi_{4}\left(3 M^{2}+1\right)+2\left(-2 \gamma^{2}+2 \gamma+1\right) M^{4}+\left(-2 \gamma^{2}-3 \gamma+3\right) M^{2}-\gamma+1\right]\right. \\
& +\pi_{2}\left\{\pi_{3} \pi_{4}\left[\left(-8 \gamma^{2}+7 \gamma+3\right) M^{4}-4\left(\gamma^{2}+\gamma-2\right) M^{2}-3 \gamma+1\right]\right. \\
& \left.\left.+2\left(6 \gamma^{2}-5 \gamma+1\right) M^{6}+\left(-\gamma^{2}+28 \gamma-11\right) M^{4}+2\left(-2 \gamma^{2}+\gamma+7\right) M^{2}+\gamma^{2}-4 \gamma+3\right\}\right),
\end{aligned}
$$

where

$$
\begin{aligned}
\pi_{1} & =\left[\left(9 \gamma^{3}-13 \gamma^{2}+11 \gamma+1\right) M^{6}+\left(-7 \gamma^{3}+35 \gamma^{2}-53 \gamma+1\right) M^{4}\right. \\
& \left.+\left(3 \gamma^{3}-7 \gamma^{2}+73 \gamma-13\right) M^{2}-\gamma^{3}-3 \gamma^{2}-19 \gamma+15\right]^{1 / 2} \\
\pi_{2} & =\left[(\gamma+1)\left(M^{2}-1\right)\right]^{1 / 2} \\
\pi_{3} & =\left[2+(\gamma-1) M^{2}\right]^{1 / 2} \\
\pi_{4} & =\left[1+\gamma\left(2 M^{2}-1\right)\right]^{1 / 2}
\end{aligned}
$$

For the rarefaction-reflected case:

$$
c_{1}^{r}(M, \gamma)=\frac{2}{(\gamma+1)^{2} M^{2}} \frac{\gamma(\gamma-1) M^{6}-\left(\gamma^{2}-4 \gamma+1\right) M^{4}-3(\gamma-1) M^{2}-2}{(2 \gamma-1) M^{4}+2 M^{2}+1},
$$

which is valid when $1 \leq \gamma \leq 3$. For $\gamma>3$, the expression is very cumbersome and impractical to use. Therefore, we decide not to show here, considering that the cases with $\gamma>3$ are quite rare.

The dependence of $c_{1}^{s, r}$ on the Mach number calculated by Eqs. A.1) and (A.6) is shown by Fig. 8 for the case of $\gamma=5 / 3$. These two coefficients take similar values for this case, although the formulas are quite different. The behavior of $c_{1}^{s, r}$ is consistent with the growth velocity of the WN model around $\rho_{a 0} / \rho_{b 0}=1$ (see Fig. 6). 


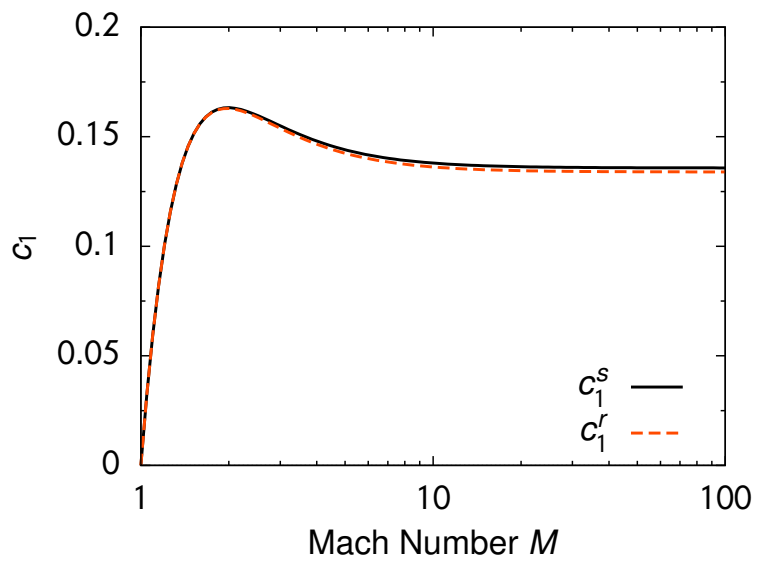

FIG. 8. Mach number dependence of the coefficients $c_{1}^{s}$ (black solid) and $c_{1}^{r}$ (red dashed) given by Eqs. (A.1) and (A.6) for the case of $\gamma=5 / 3$.

[1] S. I. Abarzhi, Phil. Trans. R. Soc. A 368, 1809 (2010).

[2] K. Nishihara, J. G. Wouchuk, C. Matsuoka, R. Ishizaki, and V. V. Zhakhovsky, Phil. Trans. R. Soc. A 368, 1769 (2010).

[3] R. D. Richtmyer, Commun. Pure Appl. Math. 13, 297 (1960).

[4] E. E. Meshkov, Fluid Dyn. 4, 101 (1969).

[5] K. A. Meyer and P. J. Blewett, Phys. Fluids 15, 753 (1972)

[6] K. O. Mikaelian, Phys. Rev. Lett. 71, 2903 (1993)

[7] Y. Yang, Q. Zhang, and D. H. Sharp, Phys. Fluids 6, 1856 (1994).

[8] J. G. Wouchuk and K. Nishihara, Phys. Plasmas 3, 3761 (1996).

[9] J. G. Wouchuk and K. Nishihara, Phys. Plasmas 4, 1028 (1997).

[10] M. Vandenboomgaerde, C. Mügler, and S. Gauthier, Phys. Rev. E 58, 1874 (1998)

[11] Q. Zhang and S.-I. Sohn, Phys. Fluids 9, 1106 (1997).

[12] O. Sadot, L. Erez, U. Alon, D. Oron, L. A. Levin, G. Erez, G. Ben-Dor, and D. Shvarts, Phys. Rev. Lett. 80, 1654 (1998)

[13] C. Matsuoka, K. Nishihara, and Y. Fukuda, Phys. Rev. E 67, 036301 (2003).

[14] M. Latini, O. Schilling, and W. S. Don, Phys. Fluids 19, 024104 (2007)

[15] G. Dimonte and P. Ramaprabhu, Phys. Fluids 22, 014104 (2010).

[16] J. W. Jacobs and J. M. Sheeley, Phys. Fluids 8, 405 (1996)

[17] M. Brouillette and R. Bonazza, Phys. Fluids 11, 1127 (1999).

[18] P. R. Chapman and J. W. Jacobs, Phys. Fluids 18, 074101 (2006).

[19] G. Dimonte and B. Remington, Phys. Rev. Lett. 70, 1806 (1993).

[20] D. R. Farley, T. A. Peyser, L. M. Logory, S. D. Murray, and E. W. Burke, Phys. Plasmas 6, 4304 (1999).

[21] S. G. Glendinning, J. Bolstad, D. G. Braun, M. J. Ed- wards, W. W. Hsing, B. F. Lasinski, H. Louis, A. Miles, J. Moreno, T. A. Peyser, B. A. Remington, H. F. Robey, E. J. Turano, C. P. Verdon, and Y. Zhou, Phys. Plasmas 10, 1931 (2003).

[22] Y. Aglitskiy, N. Metzler, M. Karasik, V. Serlin, A. L. Velikovich, S. P. Obenschain, A. N. Mostovych, A. J. Schmitt, J. Weaver, J. H. Gardner, and T. Walsh, Phys. Plasmas 13, 080703 (2006)

[23] C. F. McKee and J. P. Ostriker, Astrophys. J. 218, 148 (1977),

[24] S. Atzeni and J. Meyer-ter-Vehn, The Physics of Inertial Fusion: Beam Plasma Interaction, Hydrodynamics, Hot Dense Matter (Clarendon Press, Oxford, 2004).

[25] R. Betti and O. A. Hurricane, Nature Phys. 12, 435 (2016)

[26] T. Inoue, R. Yamazaki, and S. Inutsuka, Astrophys. J. 695, 825 (2009).

[27] T. Sano, K. Nishihara, C. Matsuoka, and T. Inoue, Astrophys. J. 758, 126 (2012).

[28] R. Samtaney, Phys. Fluids 15, L53 (2003).

[29] V. Wheatley, D. I. Pullin, and R. Samtaney, Phys. Rev. Lett. 95, 125002 (2005).

[30] T. Sano, T. Inoue, and K. Nishihara, Phys. Rev. Lett. 111, 205001 (2013).

[31] S. Chandrasekhar, Hydrodynamic and Hydromagnetic Stability (Dover, New York, 1961).

[32] J. M. Stone and T. Gardiner, Astrophys. J. 671, 1726 (2007).

[33] F. Cobos Campos and J. G. Wouchuk,

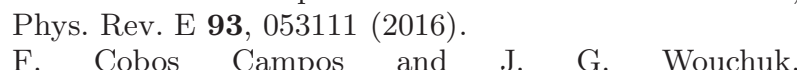
Phys. Rev. E 96, 013102 (2017).

[35] D. H. Munro, Phys. Rev. A 38, 1433 (1988).

[36] K. O. Mikaelian, Phys. Rev. A 31, 410 (1985)

[37] R. I. Klein, C. F. McKee, and P. Colella, Astrophys. J. 420, 213 (1994).

[38] F. Nakamura, C. F. McKee, R. I. Klein, and R. T. Fisher, Astrophys. J. Suppl. 164, 477 (2006)

[39] S. A. E. G. Falle, B. Vaidya, and T. W. Hartquist, 
Mon. Not. R. Astron. Soc. 465, 260 (2017)

[40] H. Takabe, K. Mima, L. Montierth, and R. L. Morse, Phys. Fluids 28, 3676 (1985).

[41] R. Betti, V. N. Goncharov, R. L. McCrory, and C. P. Verdon, Phys. Plasmas 5, 1446 (1998)

[42] G. Fraley, Phys. Fluids 29, 376 (1986).

[43] A. L. Velikovich, Phys. Fluids 8, 1666 (1996)

[44] J. G. Wouchuk, Phys. Rev. E 63, 056303 (2001).

[45] J. G. Wouchuk, Phys. Plasmas 8, 2890 (2001).

[46] B. van Leer, J. Comp. Phys. 32, 101 (1979).
[47] T. Sano, S. Inutsuka, and S. M. Miyama, Astrophys. J. Lett. 506, L57 (1998).

[48] P. Colella and P. R. Woodward, J. Comp. Phys. 54, 174 (1984).

[49] T. Hanawa, H. Mikami, and T. Matsumoto, J. Comp. Phys. 227, 7952 (2008).

[50] J. G. Wouchuk and T. Sano, Phys. Rev. E 91, 023005 (2015). 